\title{
OPTIMAL RETENTION FOR A STOP-LOSS REINSURANCE UNDER THE VaR AND CTE RISK MEASURES
}

\author{
BY \\ Jun Cai and Ken Seng Tan
}

\begin{abstract}
We propose practical solutions for the determination of optimal retentions in a stop-loss reinsurance. We develop two new optimization criteria for deriving the optimal retentions by, respectively, minimizing the value-at-risk (VaR) and the conditional tail expectation (CTE) of the total risks of an insurer. We establish necessary and sufficient conditions for the existence of the optimal retentions for two risk models: individual risk model and collective risk model. The resulting optimal solution of our optimization criterion has several important characteristics: (i) the optimal retention has a very simple analytic form; (ii) the optimal retention depends only on the assumed loss distribution and the reinsurer's safety loading factor; (iii) the CTE criterion is more applicable than the VaR criterion in the sense that the optimal condition for the former is less restrictive than the latter; (iv) if optimal solutions exist, then both VaR- and CTE-based optimization criteria yield the same optimal retentions. In terms of applications, we extend the results to the individual risk models with dependent risks and use multivariate phase type distribution, multivariate Pareto distribution and multivariate Bernoulli distribution to illustrate the effect of dependence on optimal retentions. We also use the compound Poisson distribution and the compound negative binomial distribution to illustrate the optimal retentions in a collective risk model.
\end{abstract}

\section{KEYWORDS}

Stop-loss reinsurance; expected value principle; retention; loading factor; valueat-risk (VaR); conditional tail expectation (CTE); multivariate phase type distribution; multivariate Pareto distribution; individual risk model; collective risk model.

\section{INTRODUCTION}

Reinsurance is a mechanism of transferring risk from an insurer to a second insurance carrier. The former party is referred to as the cedent (or simply the insurer) while the latter is the reinsurer. Reinsurance provides an opportunity 
for the insurer to reduce the underwriting risk and hence leads to a more effective management of risk. The stop-loss, the excess-of-loss, and the quota-share are some examples of reinsurance designs. For a fixed reinsurance premium, it is well-known that the stop-loss contract is the optimal solution among a wide arrays of reinsurance in the sense that it gives the smallest variance of the insurer's retained risk. See, for example, Bowers, et al. (1997), Daykin, et al. (1994), and Kaas, et al. (2001). This paper contributes to the optimal reinsurance research by proposing new optimization criteria using recently proposed risk measures. Analytical solutions to the retention limit of a stop-loss reinsurance are derived.

We now introduce some notations and provide the necessary background. Let $X$ be the (aggregate) loss for an insurance portfolio or an insurer. We assume that $X$ is a nonnegative random variable with cumulative distribution function $F_{X}(x)=\operatorname{Pr}\{X \leq x\}$, survival function $S_{X}(x)=\operatorname{Pr}\{X>x\}$, and mean $\mathrm{E}[X]>0$. Furthermore, we let $X_{I}$ and $X_{R}$ be, respectively, the loss random variables of the cedent and the reinsurer in the presence of a stop-loss reinsurance. Then $X_{I}$ and $X_{R}$ are related to $X$ as follows:

$$
X_{I}=\left\{\begin{array}{ll}
X, & X \leq d \\
d, & X>d
\end{array}=X \wedge d\right.
$$

and

$$
X_{R}=\left\{\begin{array}{ll}
0, & X \leq d \\
X-d, & X>d
\end{array}=(X-d)_{+},\right.
$$

where the parameter $d>0$ is known as the retention, $a \wedge b=\min \{a, b\}$, and $(a)_{+}=\max \{a, 0\}$. Under stop-loss agreement, the reinsurer pays part of $X$ that exceeds the retention limit. This implies the reinsurer absorbs the risk that exceeds the retention limit while the insurer is effectively protected from a potential large loss by limiting the liability to the retention level.

In exchange of undertaking the risk, the reinsurer charges a reinsurance premium to the cedent. A number of premium principles have been proposed for determining the appropriate level of the premium. One of the commonly used principles is the expected value principle in which the reinsurance premium, $\delta(d)$, is determined by $\delta(d)=(1+\rho) \pi(d)$, where $\rho>0$ is known as the relative safety loading and

$$
\pi(d)=\mathrm{E}\left[X_{R}\right]=\mathrm{E}\left[(X-d)_{+}\right]=\int_{d}^{\infty} S_{X}(x) d x
$$

is the (net) stop-loss premium. See, for example, Cai (2004) and Klugman et al. (2004). Naturally, the reinsurance premium $\delta(d)$ is a decreasing function of $d$. By $T$ we denote the total cost of the insurer in the presence of the stop-loss reinsurance. The total cost $T$ is captured by two components: the retained loss and the reinsurance premium; that is,

$$
T=X_{I}+\delta(d)
$$


The relation above demonstrates the classic trade-off between the risk assumed by the insurer and the risk transferred to the reinsurer. If the retention $d$ is small, then the retained liability to the cedent is expected to be low but at the expense of the higher premium payable to the reinsurer. On the other hand, if the cedent were to reduce the cost of the reinsurance premium by raising $d$, then the cedent is exposed to a potentially large liability. Consequently, determining an optimal level of retention $d$ is important to the cedent. There are many ways of determining the optimal retention $d$ depending on the chosen criterion. For example, we can select $d$ that optimally minimizes ruin probability of an insurer or optimally maximizes utility of an insurer. See, for instance, Centeno (2002, 2004). By exploiting the recently introduced value-at-risk (VaR) and conditional tail expectation (CTE) risk measures, this paper proposes a framework that optimally determines the retention limit of the stop-loss reinsurance.

Risk measures such as VaR and CTE have generated tremendous interests among practitioners and academicians. They are used extensively within banking and insurance sectors for quantifying market risks, portfolio optimization, setting capital adequacy, etc.; see for example, Jorion (2000), Krokhmal, Palmquist and Uryasev (2002), Cai and Li (2005a).

Formally, the VaR of a random variable $X$ at a confidence level $1-\alpha, 0<$ $\alpha<1$, is defined as $\operatorname{VaR}_{X}(\alpha)=\inf \{x: \operatorname{Pr}\{X>x\} \leq \alpha\}=\inf \{x: \operatorname{Pr}\{X \leq x\} \geq 1-\alpha\}$. Equivalently, it corresponds to the $100(1-\alpha)$ th percentile of $X$. Hence, $\operatorname{Pr}\{X>$ $\left.\operatorname{VaR}_{X}(\alpha)\right\} \leq \alpha$ while for any $x<\operatorname{VaR}_{X}(\alpha), \operatorname{Pr}\{X>x\}>\alpha$.

If $X$ has a one-to-one continuous distribution function on $[0, \infty)$, then $\operatorname{VaR}_{X}(\alpha)$ is the unique solution to either of the following two equations

$$
\begin{aligned}
& \operatorname{Pr}\left\{X>\operatorname{VaR}_{X}(\alpha)\right\}=\alpha, \\
& \operatorname{Pr}\left\{X \leq \operatorname{VaR}_{X}(\alpha)\right\}=1-\alpha,
\end{aligned}
$$

or more compactly as $\operatorname{VaR}_{X}(\alpha)=S_{X}^{-1}(\alpha)=F_{X}^{-1}(1-\alpha)$, where $S_{X}^{-1}$ and $F_{X}^{-1}$ are the inverse functions of $S_{X}$ and $F_{X}$, respectively.

The VaR measure has the advantage of its simplicity. If we know the corresponding VaR of a risk, then we are assured that the probability of the risk exceeding such a value is no greater than $\alpha$. In this regard, the parameter $\alpha$ can be interpreted as the risk tolerance probability. In practice, $\alpha$ is often selected to be a small value such as less than 5\%. The downside of this measure is that it provides no information on the severity of the shortfall for the risk beyond the threshold. Furthermore, some researchers advocate the importance of a coherent risk measure and the VaR is one that fails to satisfy the axiomatic properties of coherence.

We now turn to another risk measure known as the conditional tail expectation (CTE). According to Artzner et al. (1999) and Wirch and Hardy (1999), the CTE of a random variable $X$ at its $\operatorname{VaR}_{X}(\alpha)$ is formally defined as

$$
\mathrm{CTE}_{X}(\alpha)=\mathrm{E}\left[X \mid X>\operatorname{VaR}_{X}(\alpha)\right]
$$


or

$$
\operatorname{CTE}_{X}(\alpha)=\mathrm{E}\left[X \mid X \geq \operatorname{VaR}_{X}(\alpha)\right]
$$

Note that when $X$ is a continuous random variable, both (1.5) and (1.6) are identical. Furthermore, it is easy to see that $\operatorname{CTE}_{X}(\alpha) \geq \operatorname{VaR}_{X}(\alpha)$ holds for either (1.5) and (1.6). The CTE is intuitively appealing in that it captures the expected magnitude of loss given that risk exceeds or equal to its VaR. More importantly under suitable conditions, say that risks are continuous, CTE is a coherent risk measure.

Analogously, we can define VaR and CTE in terms of the insurer's retained loss $X_{I}$ and the insurer's total cost $T$. For $\operatorname{VaR}$, we have $\operatorname{VaR}_{X_{I}}(d, \alpha)=\inf \{x$ : $\left.\operatorname{Pr}\left\{X_{I}>x\right\} \leq \alpha\right\}$ and $\operatorname{VaR}_{T}(d, \alpha)=\inf \{x: \operatorname{Pr}\{T>x\} \leq \alpha\}$. Similarly for CTE, we have

$$
\mathrm{CTE}_{X_{I}}(d, \alpha)=\mathrm{E}\left[X_{I} \mid X_{I} \geq \operatorname{VaR}_{X_{I}}(d, \alpha)\right]
$$

and

$$
\mathrm{CTE}_{T}(d, \alpha)=\mathrm{E}\left[T \mid T \geq \operatorname{VaR}_{T}(d, \alpha)\right]
$$

Note that we have explicitly introduced an argument $d$ to the above VaR and CTE notations to emphasize that these risk measures are functions of the retention limit $d$. Also for $d>0$, we use only (1.6) to define the CTE counterparts for $X_{I}$ and $T$. We will demonstrate later that some values of $d$, (1.5) is not be appropriate for $T$ and $X_{I}$.

From an insurer's point of view, a prudent risk management is to ensure that the risk measures associated with $T$ are as small as possible. This motivates us to consider the following two optimization criteria for seeking the optimal level of retention. The first approach determines the optimal retention $d$ by minimizing the corresponding VaR; i.e.,

$$
\text { VaR-optimization: } \operatorname{VaR}_{T}\left(d^{*}, \alpha\right)=\min _{d>0}\left\{\operatorname{VaR}_{T}(d, \alpha)\right\}
$$

The resulting optimal retention $d^{*}$ ensures that the VaR of the total cost is minimized for a given risk tolerance level $\alpha$. We refer this method as the VaR-optimization. The second approach, which we denote as the CTE-optimization, is to determine the optimal retention $\tilde{d}$ that minimizes the CTE as shown below:

$$
\text { CTE-optimization: } \operatorname{CTE}_{T}(\tilde{d}, \alpha)=\min _{d>0}\left\{\mathrm{CTE}_{T}(d, \alpha)\right\} \text {. }
$$

The optimal retention $\tilde{d}$ from the above optimization has the appealing feature that focuses on the right tail risk by minimizing the expected loss of the extreme events.

We now provide an alternate justification of the VaR-based optimization from the point of view of a minimum capital requirement. By assuming risk $X$, 
the insurer charges an insurance premium $p_{X}$ to the insured and at the same time sets aside a minimum capital $r_{X}$ so that the insurer's probability of insolvency is at most $\alpha$. In other words, given $\alpha$ and $p_{X}$, the minimum capital $r_{X}$ is the solution to the following inequality:

$$
\operatorname{Pr}\left\{T>r_{X}+p_{X}\right\} \leq \alpha .
$$

In practice insurer prefers to set aside as little capital as possible while satisfying the insolvency constraint. From the definition of $\operatorname{VaR}_{T}(d, \alpha)$, we immediately have the following relationship:

$$
r_{X}=\operatorname{VaR}_{T}(d, \alpha)-p_{X} .
$$

The linear relation between $r_{X}$ and $\operatorname{VaR}_{T}(d, \alpha)$ implies that if $d^{*}$ is the optimal solution to (1.9), then the capital requirement is also minimized at the insolvency constraint.

In this paper, we also extend our results by considering two classes of risk models: the individual risk models and the collective risk models. In an individual risk model, the aggregate loss is given by $X=X_{1}+\cdots+X_{n}$, where $X_{j}$ corresponds to the loss in subportfolio $j$ or event $j$, for $j=1, \ldots, n$. In a collective risk model, the aggregate loss is denoted by $X=\sum_{j=1}^{N} X_{j}$, where the random variable $N$ denotes the number of losses and $X_{j}$ is the severity of the $j$ th loss, for $j=1,2, \ldots$

To encompass these two models, we assume throughout this paper that $X$ has a one-to-one continuous distribution function on $(0, \infty)$ with a possible jump at 0 and $S_{X}^{-1}(x)$ exists for $0<x<S_{X}(0)$. Furthermore, we denote $S_{X}^{-1}(0)=\infty$ and $S_{X}^{-1}(x)=0$ for $S_{X}(0) \leq x \leq 1$. We also enforce the condition $0<\alpha<S_{X}(0)$; otherwise for $\alpha \geq S_{X}(0)$, we have a trivial case since $\operatorname{VaR}_{X}(\alpha)=0$ and $\operatorname{VaR}_{X_{I}}(d, \alpha)=0$. Note that $S_{X}(0)=1$ when the distribution function of $X$ is continuous at 0 .

The rest of the paper is organized as follows. Sections 2 and 3 present, respectively, the optimal solutions as well as the conditions for which the optimal retention exists for the VaR-and CTE-optimization. Section 4 applies the general results of Sections 2 and 3 to individual risk models with dependent risks. The optimal retentions and the effect of dependence on the optimal retentions are analyzed by examining three special cases: a multivariate phasetype distribution, a multivariate Pareto distribution and a multivariate Bernoulli distribution. Section 5 applies the general results to a collective risk model by considering two special cases: compound Poisson and compound negative binomial distributions. Section 6 concludes the paper and Appendix collects the proofs of our main results.

\section{OPTIMAL RETENTION: VAR-OPTIMIZATION}

In this section, we analyze the optimal solution to the VaR-optimization (1.9). First note that the survival function of the retained loss $X_{I}$ is given by 


$$
S_{X_{I}}(x)= \begin{cases}S_{X}(x), & 0 \leq x<d \\ 0, & x \geq d .\end{cases}
$$

From the above results, if $0<\alpha \leq S_{X}(d)$ or equivalently $0<d \leq S_{X}^{-1}(\alpha)$, then $\operatorname{VaR}_{X_{I}}(d, \alpha)=d$; if $\alpha>S_{X}(d)$ or equivalently $d>S_{X}^{-1}(\alpha)$, then $\operatorname{VaR}_{X_{I}}(d, \alpha)=S_{X}^{-1}(\alpha)$. Hence, the VaR of the retained loss $X_{I}$ can be represented as

$$
\operatorname{VaR}_{X_{I}}(d, \alpha)= \begin{cases}d, & 0<d \leq S_{X}^{-1}(\alpha), \\ S_{X}^{-1}(\alpha), & d>S_{X}^{-1}(\alpha) .\end{cases}
$$

We point out that given $d>0, \operatorname{VaR}_{X_{I}}(d, \alpha)=d$ is the same for all $\alpha \in\left(0, S_{X}(d)\right]$ since $X_{I}$ is a bounded random variable with $0 \leq X_{I} \leq d$.

It follows immediately from (1.2) that there exists we a simple relationship between the $\mathrm{VaR}$ of the total cost and the $\mathrm{VaR}$ of the retained risk:

$$
\operatorname{VaR}_{T}(d, \alpha)=\operatorname{VaR}_{X_{I}}(d, \alpha)+\delta(d) .
$$

Observe that $\operatorname{VaR}_{X_{I}}(d, \alpha)$ is an increasing function of $d$ while $\delta(d)$ is a decreasing function of $d$. By combining both (2.2) and (2.3), we obtain an expression for $\operatorname{VaR}_{T}(d, \alpha)$ which we summarize in the following proposition:

Proposition 2.1 For each $d>0$ and $0<\alpha<S_{X}(0)$,

$$
\operatorname{VaR}_{T}(d, \alpha)= \begin{cases}d+\delta(d), & 0<d \leq S_{X}^{-1}(\alpha), \\ S_{X}^{-1}(\alpha)+\delta(d), & d>S_{X}^{-1}(\alpha)\end{cases}
$$

Note that similar to $\operatorname{VaR}_{X_{I}}(d, \alpha)$, the $\operatorname{VaR}$ of $T$, for a given $d>0$, is the same for all $\alpha \in\left(0, S_{X}(d)\right]$.

We now present the key result of this section. It is convenient to first define

$$
\rho^{*}=\frac{1}{1+\rho}
$$

which plays a critical role in the solutions to our optimization problems. The following theorem states the necessary and sufficient conditions for the existence of the optimal retention of the VaR-optimization (1.9):

Theorem 2.1 (a) The optimal retention $d^{*}>0$ in (1.9) exists if and only if both

and

$$
\alpha<\rho^{*}<S_{X}(0)
$$

$$
S_{X}^{-1}(\alpha) \geq S_{X}^{-1}\left(\rho^{*}\right)+\delta\left(S_{X}^{-1}\left(\rho^{*}\right)\right)
$$

hold. 
(b) When the optimal retention $d^{*}$ in (1.9) exists, then $d^{*}$ is given by

$$
d^{*}=S_{X}^{-1}\left(\rho^{*}\right)
$$

and the minimum VaR of $T$ is given by

$$
\operatorname{VaR}_{T}\left(d^{*}, \alpha\right)=d^{*}+\delta\left(d^{*}\right) .
$$

See Appendix for the proof of the above theorem.

Remark 2.1 We emphasize some practical significance of the above results. First, conditions (2.5) and (2.6) are relatively easy to verify. Second, the optimal retention is explicit and easy to compute. Third, it is of interest to note that the optimal retention, if it exists, depends only on the assumed loss distribution and the reinsurer's loading factor.

The following corollary gives the sufficient condition for the existence of the optimal retention in (1.9). See Appendix for the proof. This result provides a simple way of verifying if the optimal retention in VaR-optimization (1.9) exists.

Corollary 2.1 The optimal retention $d^{*}>0$ in (1.9) exists if both (2.5) and

$$
S_{X}^{-1}(\alpha) \geq(1+\rho) \mathrm{E}[X]
$$

hold; and the optimal retention $d^{*}$ and the minimum VaR are given by (2.7) and (2.8), respectively.

We now provide two examples to illustrate the results we just established.

Example 2.1 Assume $\alpha=0.1, \rho=0.2$ and $X$ is exponentially distributed with mean $\mathrm{E}[X]=1,000$. Note that $S_{X}(x)=e^{-0.001 x}, x \geq 0 ; S_{X}^{-1}(x)=-1,000 \log x, 0<$ $x<1$; and $S_{X}(0)=1$. Furthermore, both conditions (2.5) and (2.9) are satisfied since $\rho^{*}=0.8 \dot{3}>\alpha=0.1$ and $S_{X}^{-1}(\alpha)=-1,000 \log \alpha=2302.59>(1+\rho) \mathrm{E}[X]=1,200$. By Corollary 2.1, the optimal retention $d^{*}$ exists and equals to $d^{*}=S_{X}^{-1}\left(\rho^{*}\right)=$ $1,000 \log (1+\rho)=182.32$.

Example 2.2 Similar to Example 2.1, we consider $\alpha=0.1$ and $\rho=0.2$ except that $X$ has a Pareto distribution with $S_{X}(x)=\left(\frac{2,000}{x+2,000}\right)^{3}, x \geq 0$. Then $S_{X}^{-1}(x)=$ $2,000 x^{-1 / 3}-2,000,0<x<1$, so that $\rho^{*}=0.8 \dot{3}>\alpha=0.1$ and $S_{X}^{-1}(\alpha)=2,000 \alpha^{-1 / 3}$ $2,000=2308.87>(1+\rho) \mathrm{E}[X]=1,200$. Hence conditions (2.5) and (2.9) are satisfied with the optimal retention equals to $d^{*}=S_{X}^{-1}\left(\rho^{*}\right)=125.32$.

Remark 2.2 Note that the parameter values in the above two examples are selected so that the risks $X$ in both cases have the same mean. The Pareto distribution, 
on the other hand, is heavy-tailed. This implies that larger loss is more likely with the Pareto distribution than with the exponential distribution. Consequently, the optimal retention for the Pareto case should be smaller than the exponential case, as confirmed by our examples.

\section{Optimal Retention: CTE-OPTIMIZATION}

We now consider the optimal retention for the CTE-optimization (1.10). It follows from (1.2), (1.8) and (2.3) that the CTE of the total cost $T$ can be decomposed as:

$\mathrm{CTE}_{T}(d, \alpha)=\mathrm{E}\left[X_{I}+\delta(d) \mid X_{I}+\delta(d) \geq \operatorname{VaR}_{T}(d, \alpha)\right]=\mathrm{CTE}_{X_{I}}(d, \alpha)+\delta(d)$.

Furthermore, (1.7) implies that

$$
\begin{aligned}
\operatorname{CTE}_{X_{I}}(d, \alpha) & =\mathrm{E}\left[\operatorname{VaR}_{X_{I}}(d, \alpha)+X_{I}-\operatorname{VaR}_{X_{I}}(d, \alpha) \mid X_{I} \geq \operatorname{VaR}_{X_{I}}(d, \alpha)\right] \\
& =\operatorname{VaR}_{X_{I}}(d, \alpha)+\frac{\int_{\operatorname{VaR}_{X_{I}}(d, \alpha)}^{\infty} S_{X_{I}}(x) d x}{\operatorname{Pr}\left\{X_{I} \geq \operatorname{VaR}_{X_{I}}(d, \alpha)\right\}}
\end{aligned}
$$

It follows from $0<\operatorname{VaR}_{X_{I}}(d, \alpha) \leq d$, (2.1) and (2.2) that

$$
\begin{aligned}
\int_{\operatorname{VaR}_{X_{I}}(d, \alpha)}^{\infty} S_{X_{I}}(x) d x & =\int_{\operatorname{VaR}_{X_{I}}(d, \alpha)}^{\infty} S_{X}(x) d x \\
& = \begin{cases}0, & 0<d \leq S_{X}^{-1}(\alpha) \\
\int_{S_{X}^{-1}(\alpha)}^{d} S_{X}(x) d x, & d>S_{X}^{-1}(\alpha)\end{cases}
\end{aligned}
$$

and

$$
\begin{aligned}
\operatorname{Pr}\left\{X_{I} \geq \operatorname{VaR}_{X_{I}}(d, \alpha)\right\}=\operatorname{Pr}\left\{X_{I}=\operatorname{VaR}_{X_{I}}(d, \alpha)\right\}+S_{X_{I}}\left(\operatorname{VaR}_{X_{I}}(d, \alpha)\right) \\
= \begin{cases}\operatorname{Pr}\left\{X_{I}=d\right\}+S_{X_{I}}(d), & 0<d \leq S_{X}^{-1}(\alpha) \\
\operatorname{Pr}\left\{X_{I}=S_{X}^{-1}(\alpha)\right\}+S_{X_{I}}\left(S_{X}^{-1}(\alpha)\right), & d>S_{X}^{-1}(\alpha)\end{cases} \\
= \begin{cases}\operatorname{Pr}\{X \geq d\}, & 0<d \leq S_{X}^{-1}(\alpha), \\
S_{X}\left(S_{X}^{-1}(\alpha)\right)=\alpha, & d>S_{X}^{-1}(\alpha) .\end{cases}
\end{aligned}
$$

Thus, by combining (3.1)-(3.4) and (2.4), we derive an expression for $\mathrm{CTE}_{T}(d, \alpha)$ as follows: 
Proposition 3.1 For each $d>0$ and $0<\alpha<S_{X}(0)$,

$$
\mathrm{CTE}_{T}(d, \alpha)= \begin{cases}d+\delta(d), & 0<d \leq S_{X}^{-1}(\alpha), \\ S_{X}^{-1}(\alpha)+\delta(d)+\frac{1}{\alpha} \int_{S_{X}^{-1}(\alpha)}^{d} S_{X}(x) d x, & d>S_{X}^{-1}(\alpha) .\end{cases}
$$

Remark 3.1 Similar to the observation we made earlier, given $d>0$ the $\mathrm{CTE}_{T}(d, \alpha)$ is the same for all $\alpha \in\left(0, S_{X}(d)\right]$.

We also remark that if $0<d \leq S_{X}^{-1}(\alpha)$, then $\operatorname{VaR}_{X_{I}}(d, \alpha)=d$ and $\operatorname{VaR}_{T}(d, \alpha)=$ $d+\delta(d)$. Hence, in this case, $X_{I}>\operatorname{VaR}_{X_{I}}(d, \alpha)$ and $T>\operatorname{VaR}_{T}(d, \alpha)$ do not hold since $0 \leq X_{I} \leq d$ and $0 \leq T \leq d+\delta(d)$. Therefore, (1.5) is not appropriate for $T$ and $X_{I}$ in this case. This is why we adopt (1.6) for $T$ and $X_{I}$ in this paper. Note that for $0<d \leq S_{X}^{-1}(\alpha), \operatorname{CTE}_{T}(d, \alpha)=\operatorname{VaR}_{T}(d, \alpha)$ while for $d>S_{X}^{-1}(\alpha), \operatorname{CTE}_{T}(d, \alpha)>$ $\operatorname{VaR}_{T}(d, \alpha)$.

Now, we are ready to discuss the existence of the optimal retention for the optimization problem (1.10) based on the CTE of the total cost. The following theorem (with the proof in the Appendix) states the necessary and sufficient conditions for the existence of the optimal retention to the CTE-optimization (1.10).

Theorem 3.1 (a) The optimal retention $\tilde{d}>0$ in (1.10) exists if and only if

$$
0<\alpha \leq \rho^{*}<S_{X}(0) .
$$

(b) When the optimal retention $\tilde{d}>0$ in (1.10) exists, then

$$
\tilde{d}=S_{X}^{-1}\left(\rho^{*}\right) \text { if } \alpha<\rho^{*},
$$

and

$$
\tilde{d} \geq S_{X}^{-1}\left(\rho^{*}\right) \text { if } \alpha=\rho^{*},
$$

Because the total cost $T$ is also bounded from above by $d+\delta(d)$ (see Remark 3.1), the observations that we made in Remark 2.1 for the VaR-optimization are equally applicable to the present model.

Comparing to the VaR-optimization, the optimality condition for the optimization based on CTE is less restrictive. However, it is of interest to note that both optimization criteria yield the same optimal retentions. This provides an added advantage of adopting the CTE criterion over the VaR criterion for determining the optimal retention. This point is further elaborated in the examples below.

Note also that as the reinsurer begins to charge excessively by increasing the loading factor $\rho$, this becomes progressively more expensive for the cedent 
to transfer its risk to the reinsurer. Consequently, this discourages reinsurance and forces the cedent to undertake more and more risk by raising retention level. In the limit as $\rho \rightarrow \infty$, we have $\tilde{d}=S_{X}^{-1}\left(\rho^{*}\right) \rightarrow \infty$ so that the insurer will not reinsure its risk.

We now use the following two examples to illustrate the results we just established.

Example 3.1 Assume $\alpha=0.1, \rho=2.7$ and $X$ has the same exponential distribution as in Example 2.1. Then,

$$
S_{X}^{-1}(\alpha)-\left(S_{X}^{-1}\left(\rho^{*}\right)+(1+\rho) \int_{S_{X}^{-1}\left(\rho^{*}\right)}^{\infty} S_{X}(x) d x\right)=-5.75<0 .
$$

Hence, the optimal retention $d^{*}$ does not exist since the condition (2.6) is not satisfied. However, the optimal retention $\tilde{d}$ exists since $\rho^{*}=0.27>\alpha=0.1$. Consequently by Theorem 3.1, we have $\tilde{d}=S_{X}^{-1}\left(\rho^{*}\right)=1308.33$.

Example 3.2 Similar to Example 3.1, by reconsidering Example 2.2 with $\alpha=0.1$, $\rho=2.7$, it is easy to verify that $d^{*}$ does not exist but $\tilde{d}$ exists. In this case, $\tilde{d}=$ $S_{X}^{-1}\left(\rho^{*}\right)=1093.36$, which is smaller than corresponding value in Example 3.1, as to be expected.

\section{INDIVIDUAL RISK MODEL WITH DEPENDENT RISKS}

We now consider an individual risk model consists of $n$ dependent losses (risks) $X_{1}, \ldots, X_{n}$. The aggregate loss of the portfolio is the sum of these losses, i.e., $X=X_{1}+\cdots+X_{n}$. A stop loss reinsurance with retention $d$ can similarly be written on the aggregated loss. If the distribution of $X$ is known, then the results established in the previous two sections can be used to determine the optimal retention limit.

There are several dependent models in which the distribution $X=X_{1}+\cdots+$ $X_{n}$ can be expressed analytically. We consider three particular types: a multivariate phase type distribution, a multivariate Pareto (II) distribution and a multivariate Bernoulli distribution. The effect of dependence on the optimal retentions is also analyzed.

\subsection{Dependent risks with multivariate phase type distributions}

Let $\{X(t), t \geq 0\}$ be a continuous-time and finite-state Markov chain with a finite state space $\mathcal{E}$, initial distribution vector $\boldsymbol{\beta}=(0, \boldsymbol{\alpha})$, and sub-generator

$$
Q=\left[\begin{array}{cc}
0 & 0 \\
-A e & A
\end{array}\right],
$$

where the first state in $\mathcal{E}$ denotes the absorbing state and $\boldsymbol{e}$ is a column vector of 1's. 
Let $X=\inf \{t \geq 0: X(t)=0\}$ be the time to the absorbing state in the Markov chain. Then the distribution of the random variable $X$ is said to be of phase type (PH) with representation $(\boldsymbol{\alpha}, A,|\mathcal{E}|-1)$. Denote the survival function of $X$ by $S_{X}(x)=\operatorname{Pr}\{X>x\}$. Then $X$ is of phase type with representation $(\alpha, A,|\mathcal{E}|-1)$ if and only if $S_{X}(x)=\boldsymbol{\alpha} e^{x A} \boldsymbol{e}, x \geq 0$.

A subset of the state space is said to be a closed or an absorbing subset if once the process $\{X(t), t \geq 0\}$ enters the subset, $\{X(t), t \geq 0\}$ never leaves. Let $\mathcal{E}_{i}, i=1, \ldots, n$, be $n$ closed or absorbing subsets of $\mathcal{E}$ and $X_{i}$ be the time to the absorbing subset $\mathcal{E}_{i}$, i.e. $X_{i}=\inf \left\{t \geq 0: X(t) \in \mathcal{E}_{i}\right\}, i=1, \ldots, n$. Then the joint distribution of $\left(X_{1}, \ldots, X_{n}\right)$ is called a multivariate phase type distribution $(\mathrm{MPH})$ with representation $\left(\boldsymbol{\alpha}, A, \mathcal{E}, \mathcal{E}_{1}, \ldots, \mathcal{E}_{n}\right)$, and $\left(X_{1}, \ldots, X_{n}\right)$ is called a phase type random vector. See, Assaf et al. (1984), Cai and Li (2005a, 2005b).

Examples of MPH distributions include, among many others, the well-known Marshall-Olkin distribution (Marshall and Olkin, 1967). As in the univariate case, MPH distributions (and their densities, Laplace transforms and moments) can be expressed in a closed form.

The set of $n$-dimensional MPH distributions is dense in the set of all distributions on $[0, \infty)^{n}$. Hence, any multivariate nonnegative distribution, such as multivariate lognormal distribution and multivariate Pareto distribution, can be approximated by a sequence of MPH distributions.

The distribution of the convolution of a phase type random vector is derived by Cai and $\mathrm{Li}$ (2005b) as follows.

Lemma 4.1 Let $\left(X_{1}, \ldots, X_{n}\right)$ be a PH type vector with representation $(\alpha, A, \mathcal{E}$, $\left.\mathcal{E}_{i}, i=1, \ldots, n\right)$, where $\boldsymbol{A}=\left(a_{i, j}\right)$. Then $\sum_{i=1}^{n} X_{i}$ has a phase type distribution with representation $(\boldsymbol{\alpha}, \boldsymbol{B},|\mathcal{E}|-1)$, where $\boldsymbol{B}=\left(b_{i, j}\right)$ is given by,

$$
b_{i, j}=\frac{a_{i, j}}{k(i)},
$$

where $k(i)=$ number of indexes in $\left\{j: i \notin \mathcal{E}_{j}, 1 \leq j \leq n\right\}$.

Example 4.1 Consider a two-dimensional phase type distribution with the state space $\mathcal{E}=\{12,2,1\}$, the absorbing subsets $\mathcal{E}_{j}=\{12, j\}, j=1,2$, the initial probability vector $\boldsymbol{\alpha}=(0,0,1)$, and the sub-generator $\boldsymbol{A}$

$$
\boldsymbol{A}=\left[\begin{array}{ccc}
-\lambda_{12}-\lambda_{1} & 0 & 0 \\
0 & -\lambda_{12}-\lambda_{2} & 0 \\
\lambda_{2} & \lambda_{1} & -\lambda_{12}-\lambda_{1}-\lambda_{2}
\end{array}\right] .
$$

From Lemma 4.1, the matrix $\boldsymbol{B}$ is given by

$$
\boldsymbol{B}=\left[\begin{array}{ccc}
-\lambda_{1}-\lambda_{12} & 0 & 0 \\
0 & -\lambda_{2}-\lambda_{12} & 0 \\
\frac{\lambda_{2}}{2} & \frac{\lambda_{1}}{2} & -\frac{\lambda_{12}+\lambda_{1}+\lambda_{2}}{2}
\end{array}\right] .
$$


Let $X_{1}$ and $X_{2}$ be the times to the absorbing subset $\mathcal{E}_{1}$ and $\mathcal{E}_{2}$, respectively. Thus, $\left(X_{1}, X_{2}\right)$ is a phase type random vector and the survival function of $X_{1}+X_{2}$ is given by $S_{X}(x)=\boldsymbol{\alpha} e^{x \boldsymbol{B}} \boldsymbol{e}$, where $\boldsymbol{\alpha}=(0,0,1)$ and $\boldsymbol{e}=(1,1,1)^{\prime}$.

This two-dimensional phase type distribution is also refereed as to a twodimensional Marshall-Olkin distribution (Marshall and Olkin, 1967) or the distribution of the joint-life status in a common shock model (Bowers et al., 1997).

To discuss the effect of the dependence on the optimal retentions, we assume $\alpha=0.1$ and $\rho=0.2$ as in Examples 2.1-2.2 and consider the following three cases.

Case 1: $\lambda_{12}=0, \lambda_{1}=\lambda_{2}=0.002$. In this case, $X_{1}$ and $X_{2}$ are independent, and $S_{X}(x)=(1+0.002 x) e^{-0.002 x}, x \geq 0$ is a gamma distribution. The optimal retention limit is $\tilde{d}=365.53$.

Case 2: $\lambda_{12}=0.001, \lambda_{1}=\lambda_{2}=0.001$. In this case, $X_{1}$ and $X_{2}$ are positively dependent, and $S_{X}(x)=3 e^{-0.0015 x}-2 e^{-0.002 x}, x \geq 0$ is a hyperexponential survival function. The optimal retention limit is $\tilde{d}=273.13$.

Case 3: $\lambda_{12}=0.002, \lambda_{1}=\lambda_{2}=0$. This is the comonotone case where $X_{1}=X_{2}$, and so $X_{1}$ and $X_{2}$ have the strongest positive dependence. In this case, $S_{X}(x)=e^{-0.001 x}, x \geq 0$ is an exponential survival function as that in Example 2.1. The optimal retention limit is $\tilde{d}=182.32$.

Remark 4.1 In all three cases, $\left(X_{1}, X_{2}\right)$ has the same marginal exponential distribution each with mean 500 . The only difference among them is the magnitude of the correlation between $X_{1}$ and $X_{2}$. It can be verified that the correlation coefficient between $X_{1}$ and $X_{2}$ in Case 1 is the smallest while in Case 3 is the largest. Table 1 illustrates the effect of dependence on the optimal limit $\tilde{d}$. As correlation increases; i.e. the portfolio becomes more risky, the insurer is protected by reinsuring the risk with a lower optimal retention level.

TABLE 1

Effects of Dependence on $\tilde{d}$ - Multivariate Phase Type Distribution: IN ALL CASES, WE HAVE $\mathrm{E}\left[X_{1}\right]=\mathrm{E}\left[X_{2}\right]=1000$ WITH $\alpha=0.1$ AND $\rho=0.2$

\begin{tabular}{c|c|c|c}
\hline \hline & Case 1 & Case 2 & Case 3 \\
\hline$\tilde{d}$ & 365.53 & 273.13 & 182.32 \\
\hline \hline
\end{tabular}

\subsection{Dependent risks with multivariate Pareto distributions}

Let $\left(X_{1}, \ldots, X_{n}\right)$ be a nonnegative random vector with the following joint survival function

$S\left(x_{1}, \ldots, x_{n}\right)=\operatorname{Pr}\left\{X_{1}>x_{1}, \ldots, X_{n}>x_{n}\right\}=\left(1+\sum_{i=1}^{n} \frac{x_{i}}{\sigma}\right)^{-\lambda}, x_{1} \geq 0, \ldots, x_{n} \geq 0$, 
where $\lambda>0$ and $\sigma>0$ are constants. This distribution is known as the multivariate Pareto (II) distribution. See, for example, Arnold (1983). It is easy to verify that the density function $X=X_{1}+\cdots+X_{n}$ has the following representation:

$$
f_{X}(x)=\frac{1}{\sigma B(\lambda, n)}\left(\frac{x}{\sigma}\right)^{n-1}\left(1+\frac{x}{\sigma}\right)^{-(\lambda+n)}, x \geq 0,
$$

where $B(\lambda, n)$ is the beta function. The density function (4.3) is known as the Feller-Pareto distribution (Arnold 1983).

Example 4.2 Consider a two-dimensional Pareto random vector $\left(X_{1}, X_{2}\right)$ which has a joint survival function of the form (4.2) with $n=2$ and $\lambda>2$. Hence, by (4.3), we know that $X=X_{1}+X_{2}$ has the following density function $f_{X}(x)=$ $\frac{1}{\sigma B(\lambda, 2)}\left(\frac{x}{\sigma}\right)\left(1+\frac{x}{\sigma}\right)^{-(\lambda+2)}, x \geq 0$. Furthermore, $X_{1}$ and $X_{2}$ have the same marginal Pareto distributions with $\mathrm{E}\left[X_{1}\right]=\mathrm{E}\left[X_{2}\right]=\frac{\sigma}{\lambda-1}$ and $\operatorname{Var}\left[X_{1}\right]=\operatorname{Var}\left[X_{2}\right]=$ $\frac{\lambda \sigma^{2}}{(\lambda-1)^{2}(\lambda-2)}$. Equation (6.1.29) of Arnold (1983) yields

$$
\operatorname{Cov}\left[X_{1}, X_{2}\right]=\frac{\sigma^{2}\left[\Gamma(\lambda) \Gamma(\lambda-2)-\{\Gamma(\lambda-1)\}^{2}\right]}{[\Gamma(\lambda)]^{2}}=\frac{\sigma^{2}}{(\lambda-1)^{2}(\lambda-2)} .
$$

Consequently, the correlation coefficient between $X_{1}$ and $X_{2}$ simplifies to

$$
\rho_{X_{1}, X_{2}}=\frac{\operatorname{Cov}\left[X_{1}, X_{2}\right]}{\sqrt{\operatorname{Var}\left[X_{1}\right] \operatorname{Var}\left[X_{2}\right]}}=\frac{1}{\lambda} .
$$

Table 2 depicts the optimal retention limits for the bivariate Pareto risks over three sets of parameter values. In all these cases, we assume $\alpha=0.1, \rho=0.2$ and $\left(X_{1}, X_{2}\right)$ have the same marginal Pareto distributions with mean 500. The correlation between $X_{1}$ and $X_{2}$ is the lowest for Case 1 and the highest for Case 3. Consistent with our observations in Example 4.1, the optimal retention levels with bivariate Pareto risks decrease with increasing correlation.

The above observation can be justified formally by first noticing that $B(\lambda, 2)=$ $\Gamma(\lambda) \Gamma(2) / \Gamma(\lambda+2)=1 /[\lambda(\lambda+1)]$ and that the survival function of $X$ is

$$
S_{X}(x)=\int_{x}^{\infty} \frac{1}{\sigma B(\lambda, 2)}\left(\frac{y}{\sigma}\right)\left(1+\frac{y}{\sigma}\right)^{-(\lambda+2)} d y=\left(1+\frac{x}{\sigma}\right)^{-(\lambda+1)}\left(1+\frac{x}{\sigma}+\lambda \frac{x}{\sigma}\right) .
$$

Furthermore, observe that $y<(1+y) \ln (1+y)$ for any $y>0$. Thus, for any fixed $x>0, S_{X}(x)$ is decreasing in $\lambda$ since $\frac{\vartheta S_{X}(x)}{\vartheta \lambda}<0$ and consequently the optimal retention levels with bivariate Pareto risks decrease in $\lambda$. 
TABLE 2

Effects of Dependence on $\tilde{d}$ - Multivariate Pareto Distribution: IN ALL CASES, WE HAVE $\mathrm{E}\left[X_{1}\right]=\mathrm{E}\left[X_{2}\right]=500$ WITH $\alpha=0.1$ AND $\rho=0.2$

\begin{tabular}{c|c|c|c}
\hline \hline & Case 1 & Case 2 & Case 3 \\
\hline$\lambda$ & 10 & 5 & 2.5 \\
$\sigma$ & 4,500 & 2,000 & 750 \\
$\rho_{X_{1}, X_{2}}$ & 0.1 & 0.2 & 0.4 \\
\hline$\tilde{d}=S_{X}^{-1}\left(\rho^{*}\right)$ & 324.95 & 285.89 & 211.09 \\
\hline \hline
\end{tabular}

Note also that the optimal retentions in this example are larger than that in Example 2.2. To understand this, let us first point out that the ratio of the density function in Example 2.2 to the density function in any of the three cases in this example goes to infinity as $x$ goes to infinity. This implies that the Pareto distribution in Example 2.2 asymptotically has a heavier tail than any of the three cases in this example. This again is consistent with the earlier observations that the more dangerous the risk is, the smaller the optimal retention in a stoploss reinsurance.

\subsection{Dependent risks associated with a multivariate Bernoulli distribution}

Let $\left(X_{1}, \ldots, X_{n}\right)$ be a nonnegative random vector with

$$
X_{i}=I_{i} B_{i}, \quad i=1, \ldots, n,
$$

where $B_{1}, \ldots, B_{n}$ are independent positive random variables denoting the amounts of claims and $\left(I_{1}, \ldots, I_{n}\right)$ is a multivariate Bernoulli random vector describing occurrences of claims. Furthermore, the random variables $\left\{B_{1}, \ldots, B_{n}\right\}$ are independent of the random variable $\left\{I_{1}, \ldots, I_{n}\right\}$. This is a traditional individual risk model. Another version of model (4.4) can be found in Cossette, et al. (2002). We illustrate the effects of dependence on the optimal retention in this model by setting $n=2$ in the following example.

Example 4.3 Let $n=2$ in (4.4). Assume that $B_{1}$ and $B_{2}$ are independent positive random variables with common distribution $G$, mean $\mu$, and variance $\sigma^{2}$. Furthermore, the bivariate Bernoulli random vector $\left(I_{1}, I_{2}\right)$ is distributed as

$$
\begin{array}{ll}
\operatorname{Pr}\left\{I_{1}=1, I_{2}=1\right\}=a, & \operatorname{Pr}\left\{I_{1}=1, I_{2}=0\right\}=b, \\
\operatorname{Pr}\left\{I_{1}=0, I_{2}=1\right\}=c, & \operatorname{Pr}\left\{I_{1}=0, I_{2}=0\right\}=d,
\end{array}
$$

where $0<a, b, c, d<1$ and $a+b+c+d=1$. 
Thus, the survival function of $X=X_{1}+X_{2}$ is, for $x \geq 0$,

$$
\begin{aligned}
S_{X}(x)= & \operatorname{Pr}\left\{X_{1}+X_{2}>x \mid I_{1}=1, I_{2}=1\right\} a+\operatorname{Pr}\left\{X_{1}+X_{2}>x \mid I_{1}=1, I_{2}=0\right\} b \\
& +\operatorname{Pr}\left\{X_{1}+X_{2}>x \mid I_{1}=0, I_{2}=1\right\} c+\operatorname{Pr}\left\{X_{1}+X_{2}>x \mid I_{1}=0, I_{2}=0\right\} d \\
= & a \bar{G}^{(2)}(x)+(b+c) \bar{G}(x),
\end{aligned}
$$

where $\bar{G}(x)=1-G(x), \bar{G}^{(2)}(x)=1-G^{(2)}(x)$, and $G^{(2)}(x)$ is the 2-fold convolution of $G(x)$ with itself.

It is easy to see that $\mathrm{E}\left[X_{1}\right]=\mu(a+b), \mathrm{E}\left[X_{2}\right]=\mu(a+c), \mathrm{E}\left[X_{1} X_{2}\right]=a \mu^{2}$, $\operatorname{Var}\left[X_{1}\right]=(a+b) \mathrm{E}\left[B_{1}^{2}\right]-[\mu(a+b)]^{2}=(a+b)\left[\sigma^{2}+\mu^{2}(1-(a+b))\right]$, and $\operatorname{Var}\left[X_{2}\right]=$ $(a+c)\left[\sigma^{2}+\mu^{2}(1-(a+c))\right]$. Hence,

$$
\operatorname{Cov}\left[X_{1}, X_{2}\right]=\mu^{2}[a-(a+b)(a+c)]
$$

and the correlation coefficient between $X_{1}$ and $X_{2}$ is

$$
\rho_{X_{1}, X_{2}}=\frac{\mu^{2}[a-(a+b)(a+c)]}{\sqrt{(a+b)(a+c)\left[\sigma^{2}+\mu^{2}(1-(a+b))\right]\left[\sigma^{2}+\mu^{2}(1-(a+c))\right]}} .
$$

We now consider a special case of the above model. We assume $G$ has an exponential distribution with mean $\mu=1000$ (and hence $\sigma^{2}=2 \mu^{2}$ ). We further set $\alpha=0.1, \rho=0.2$ and $a+b=a+c=0.5$ so that $\mathrm{E}\left[X_{1}\right]=\mathrm{E}\left[X_{2}\right]=500$ as in Examples 4.1 and 2.2. By considering three combinations of $a$ and $b$, the resulting optimal retentions are shown in Table 3. Note again that the higher the correlation coefficient, the lower the retention level, which is consistent with our earlier

\begin{tabular}{|c|c|c|c|}
\hline & Case 1 & Case 2 & Case 3 \\
\hline$(a, b)$ & $(0.05,0.45)$ & $(0.1,0.4)$ & $(0.15,0.35)$ \\
\hline$\rho_{X_{1}, X_{2}}$ & -0.16 & -0.12 & -0.08 \\
\hline$\tilde{d}=S_{X}^{-1}\left(\rho^{*}\right)$ & 138.28 & 86.53 & 24.04 \\
\hline
\end{tabular}
examples.

\section{TABLE 3}

EfFects of Dependence on $\tilde{d}$ - Multivariate Bernoulli model: IN ALL CASES, WE HAVE $\mathrm{E}\left[X_{1}\right]=\mathrm{E}\left[X_{2}\right]=500$ WITH $\alpha=0.1$ AND $\rho=0.2$

\section{OPTIMAL RETENTION IN A COLLECTIVE RISK MODEL}

In this section, we illustrate the results of Sections 2 and 3 by considering the collective risk model. We assume that $N, X_{1}, X_{2}, \ldots$ are independent and $X_{1}, X_{2}, \ldots$ are identically distributed. The survival function of the aggregate loss $X=\sum_{j=1}^{N} X_{j}$ can be computed via 


$$
S_{X}(x)=\sum_{n=1}^{\infty} p_{n} \bar{F}^{(n)}(x), \quad x \geq 0
$$

where $\left\{p_{n}=\operatorname{Pr}\{N=n\}, n=0,1,2, \ldots\right\}$ is the probability function of $N ; \bar{F}^{(n)}(x)=$ $1-F^{(n)}(x)$; and $F^{(n)}(x)$ is the $n$-fold convolution of the distribution function $F(x)=\operatorname{Pr}\left\{X_{j} \leq x\right\}$.

We now provide two examples to illustrate our results. The first example is a compound Poisson model and the other example is a compound negative binomial model.

Example 5.1 (Compound Poisson-Exponential Model) Suppose $N$ has a Poisson distribution with mean $\lambda>0$ and the severities of claims $\left\{X_{j} ; j=1,2, \ldots\right\}$ are i.i.d. exponential random variables with mean $\mu>0$, then

$$
S_{X}(x)=e^{-x / \mu} \int_{0}^{\lambda} e^{-s} I_{0}(2 \sqrt{x s / \mu}) d s, \quad x \geq 0
$$

where $I_{0}(z)=\sum_{j=0}^{\infty} \frac{(z / 2)^{2 j}}{(j !)^{2}}$ is a modified Bessel function of the first kind. See, for example, Seal (1969).

Now assume that $\alpha=0.1, \rho=0.2, \lambda=10$, and $\mu=100$. These parameter values yield $\mathrm{E}[X]=\lambda \mu=1,000, S_{X}(0)=1-e^{-\lambda}=0.9999546$, and $S_{X}^{-1}(0.1)=1598.27$ (using standard mathematical software), hence satisfying conditions $0<\alpha=0.1<$ $S_{X}(0)=0.9999546, \rho^{*}=0.8 \dot{3}>\alpha=0.1$, and $S_{X}^{-1}(\alpha)=1598.27>(1+\rho) \mathrm{E}[X]=1200$. By Corollary 2.1 and Theorem 3.1, the optimal solution exists for both VaR and CTE optimization criteria and both yield the same optimal retention of $S_{X}^{-1}\left(\rho^{*}\right)=569.54$.

Example 5.2 (Compound Negative Binomial-Exponential Model) Now assume that the severity distributions are the same as in the last example except that the frequency distribution $N$ follows a negative binomial with parameters $r$ and $\beta$. It can be shown (see Klugman et al. (2004)) that if $r$ is positive integer, then

$$
S_{X}(x)=\sum_{n=1}^{r}\left(\begin{array}{l}
r \\
n
\end{array}\right)\left(\frac{\beta}{1+\beta}\right)^{n}\left(\frac{\beta}{1+\beta}\right)^{r-n} \sum_{j=0}^{n-1} \frac{\left[x \mu^{-1}(1+\beta)^{-1}\right]^{j} e^{-x \mu^{-1}(1+\beta)^{-1}}}{j !} .
$$

Furthermore, $S_{X}(0)=1-(1+\beta)^{-r}$ and $\mathrm{E}[X]=r \beta \mu$. Using parameter values $\alpha=0.1, \rho=0.2, r=50, \beta=0.2$, and $\mu=100$, we obtain $\mathrm{E}[X]=1000, \rho^{*}=0.8 \dot{3}>$ $\alpha=0.1, S_{X}(0)=0.99989>0.1$, and $S_{X}^{-1}(0.1)=1628.37>(1+\rho) \mathrm{E}[X]=1200$. It follows from Corollary 2.1 and Theorem 3.1 that an optimal solution exists for both optimizations, with the optimal retention of $S_{X}^{-1}\left(\rho^{*}\right)=549.02$. Note that the optimal retention value in this case is smaller than that in the compound Poisson model. This is again to be expected since both models have the same expected number of losses and the same expected aggregated loss, which 
implies that the compound negative binomial is "more risky" than the compound Poisson in the sense that the former has a larger variance.

Remark 5.1 If we were to consider the optimal retention at $\alpha=0.35$, we would have obtained $S_{X}^{-1}(0.35)=1127.22$ for the compound Poisson example and $S_{X}^{-1}(0.35)=1130.79$ for the compound negative binomial example. These values fail to satisfy condition (2.9) and hence we can no longer use Corollary 2.1 to establish the existence of an optimal solution for the VaR-optimization. However, condition (3.6) is still valid. This implies that the optimal retentions as calculated in Examples 5.1 and 5.2 are still the corresponding optimal retentions at $\alpha=0.35$ under the CTE criterion.

To end this section, we remark that simple analytical formula for the survival function of the aggregate claims $S=X_{1}+\ldots+X_{n}$ or $S=X_{1}+\ldots X_{N}$ often does not exist. We need to resort to numerical procedure for determining the optimal retention. For instance in the above two examples, even though the survival function can be expressed in closed-form (see (5.2) and (5.3)), the optimal retentions were obtained numerically. In situation where the survival functions cannot be expressed in closed-form, one possible solution is to use normal or translated gamma approximations to approximate the survival function and then determine the optimal retention levels accordingly. See for example, Daykin et al. (1994) and Klugman et al. (2004).

\section{CONCLUSIONS}

This paper addressed the important question of determining an optimal level of retention in a stop-loss reinsurance. The proposed optimization is simple and intuitive. More importantly, the optimal retention is explicit, and can easily be calculated. If the solution exists, both CTE-optimization and VaR-optimization yield the same optimal solution. However, we argued that, in general, the CTE criterion is preferred to that based on VaR since the optimality condition is less restrictive in the former optimization framework.

We also pointed out that the safety loading and the assumed loss model are critical factors for determining the optimal retentions. If the optimal solution exists, the optimal retention as well as the minimum risk measure are the same regardless of the risk tolerance probability. We applied the results to the individual risk models with dependent risks and classical collective risk models. The effect of correlation on optimal retention was assessed. In general, the more risky the underlying risk, the lower the optimal retention.

\section{APPENDIX}

Proof of Theorem 2.1. (a) Observe that from (2.4), $\operatorname{VaR}_{T}(d, \alpha)$ is continuous on $d \in(0, \infty)$ and decreasing on $d \in\left(S_{X}^{-1}(\alpha), \infty\right)$ with the limit $S_{X}^{-1}(\alpha)$ as $d \rightarrow \infty$. Furthermore, when $\rho^{*}<S_{X}(0)$, the function $d+\delta(d)$ is decreasing for $d \in\left(0, d_{0}\right)$ 
and increasing for $d \in\left(d_{0}, \infty\right)$, where $d_{0}=S_{X}^{-1}\left(\rho^{*}\right)>0$, and hence the function $d+\delta(d)$ attains its minimum value at $d_{0}$ with minimum value $d_{0}+\delta\left(d_{0}\right)$. Consequently, if both (2.5) and (2.6) hold, then $0<d_{0}<S_{X}^{-1}(\alpha)$ and the minimum value $d_{0}+\delta\left(d_{0}\right)$ is also the global minimum value of $\operatorname{VaR}_{T}(d, \alpha)$ on $d \in(0, \infty)$. Therefore, $d_{0}>0$ is the optimal retention. See Figure 1 for a graphical representation of $\operatorname{VaR}_{T}(d, \alpha)$.

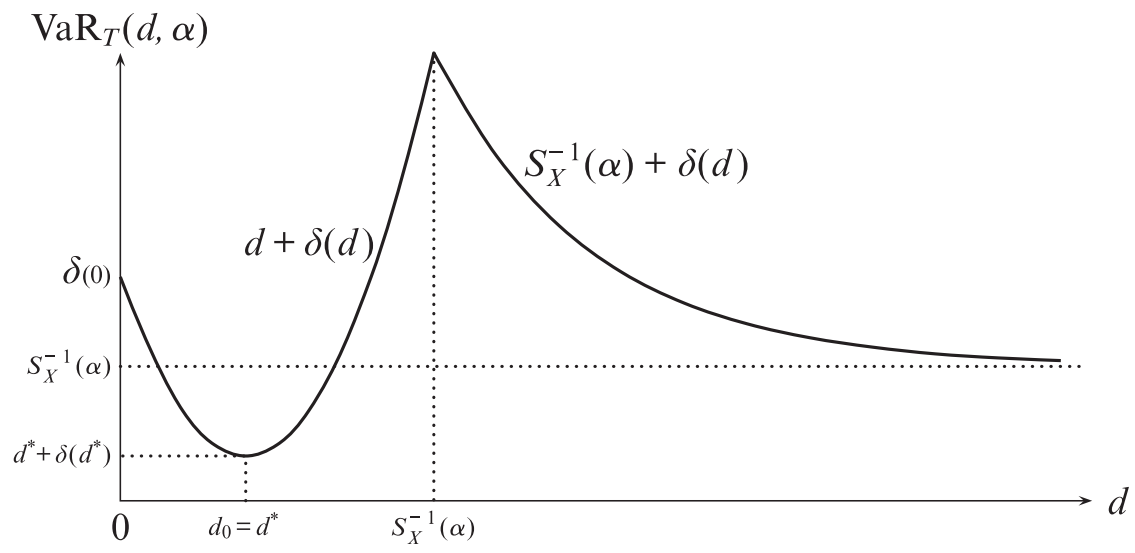

FIGURE 1: A typical graph of $\operatorname{VaR}_{T}(d, \alpha)$ if optimal retention exists.

Conversely, if (2.5) does not hold with $\alpha \geq \rho^{*}$, then $d_{0} \geq S_{X}^{-1}(\alpha)$ and $\operatorname{VaR}_{T}(d, \alpha)$ is decreasing on $d \in(0, \infty)$ with a limiting value of $S_{X}^{-1}(\alpha)$ as $d \rightarrow \infty$, hence the optimal retention $d^{*}$ does not exist; if (2.5) does not hold with $\rho^{*} \geq S_{X}(0)$, then $d_{0}=0$ and again $\operatorname{VaR}_{T}(d, \alpha)$ is decreasing on $d \in(0, \infty)$ with a limiting value of $S_{X}^{-1}(\alpha)$ as $d \rightarrow \infty$, hence the optimal retention $d^{*}$ does not exist; if (2.5) holds but (2.6) does not hold, then $\inf _{d>0} \operatorname{VaR}_{T}(d, \alpha)=S_{X}^{-1}(\alpha)$, however, no such a $d^{*}>0$ so that $\operatorname{VaR}_{T}\left(d^{*}, \alpha\right)=S_{X}^{-1}(\alpha)$. Therefore, both (2.5) and (2.6) are necessary for the optimal retention $d^{*}>0$ to exist.

(b) When the optimal retention $d^{*}>0$ exists, from the proof of (a), we have $d^{*}=d_{0}$ so that the minimum value of the $\mathrm{VaR}$ on $(0, \infty)$ is $d_{0}+\delta\left(d_{0}\right)$. Hence, (2.7) and (2.8) hold.

Proof of Corollary 2.1. It is sufficient to verify that (2.9) implies (2.6). It follows from (2.9) that

$$
\begin{aligned}
S_{X}^{-1}(\alpha) & \geq(1+\rho) \int_{0}^{S_{X}^{-1}\left(\rho^{*}\right)} S_{X}(x) d x+(1+\rho) \pi\left(S_{X}^{-1}\left(\rho^{*}\right)\right) \\
& \geq S_{X}^{-1}\left(\rho^{*}\right)+(1+\rho) \pi\left(S_{X}^{-1}\left(\rho^{*}\right)\right) .
\end{aligned}
$$

Hence (2.6) is satisfied. 
Proof of Theorem 3.1. (a) Observe that from (3.5), $\operatorname{CTE}_{T}(d, \alpha)$ is continuous on $d \in(0, \infty)$. Furthermore, when $\rho^{*}<S_{X}(0)$, the function $d+\delta(d)$ is decreasing on $d \in\left(0, d_{0}\right)$ and increasing on $d \in\left(d_{0}, \infty\right)$, where $d_{0}=S_{X}^{-1}\left(\rho^{*}\right)>0$. Taking the partial differentiation with respect to $d$, we get

$$
\frac{\partial}{\partial d}\left(S_{X}^{-1}(\alpha)+\delta(d)+\frac{1}{\alpha} \int_{S_{X}^{-1}(\alpha)}^{d} S_{X}(x) d x\right)=\left(\frac{1}{\alpha}-(1+\rho)\right) S_{X}(d) .
$$

Consequently, (3.6) implies that $0<d_{0} \leq S_{X}^{-1}(\alpha)$ and that the function $S_{X}^{-1}(\alpha)+$ $\delta(d)+\frac{1}{\alpha} \int_{S^{-1}(\alpha)}^{d} S(x) d x$ is increasing in $d$ if $\alpha<\rho^{*}$ and a constant if $\alpha=\rho^{*}$. Therefore, if $\alpha<\rho^{*}, \operatorname{CTE}_{T}(d, \alpha)$ attains its minimum value at $d_{0}=\tilde{d}=S_{X}^{-1}\left(\rho^{*}\right)$ with minimum value of $d_{0}+\delta\left(d_{0}\right)$ and hence $d_{0}>0$ is the optimal retention. Furthermore, if $\alpha=\rho^{*}, \operatorname{CTE}_{T}(d, \alpha)$ attains its minimum value at any $d=\tilde{d} \geq S_{X}^{-1}\left(\rho^{*}\right)$ with minimum value of $d_{0}+\delta\left(d_{0}\right)$. Consequently any $\tilde{d} \geq d_{0} \geq 0$ is the optimal retention in this case.

Conversely, if (3.6) does not hold with $\alpha>\rho^{*}$, then $d_{0}>S_{X}^{-1}(\alpha)$ and the function $S_{X}^{-1}(\alpha)+\delta(d)+\frac{1}{\alpha} \int_{S_{X}^{-1}(\alpha)}^{d} S_{X}(x) d x$ is decreasing. Hence, $\operatorname{CTE}_{T}(d, \alpha)$ is decreasing on $d \in(0, \infty)$ with a limiting minimum $S_{X}^{-1}(\alpha)+\frac{1}{\alpha} \int_{S_{X}^{-1}(\alpha)}^{d} S_{X}(x) d x$. The optimal retention in (1.9), therefore, does not exist; if (3.6) does not hold with $\rho^{*} \geq S_{X}(0)$, then $d_{0}=0$, there is no $\tilde{d}>0$ so that the optimal retention in (1.10) exists. This completes the proof of Theorem 3.1(a).

(b) When the optimal retention $\tilde{d}$ in (1.10) exists, it follows from the proof of (a) that $\tilde{d}=d_{0}=S_{X}^{-1}\left(\rho^{*}\right)$ if $\alpha<\rho^{*}$, and $\tilde{d} \geq S_{X}^{-1}\left(\rho^{*}\right)$ if $\alpha=\rho^{*}$.

\section{ACKNOWLEDGMENT}

Cai acknowledges research support from the Natural Sciences and Engineering Research Council of Canada and the Canada Foundation for Innovation. Tan thanks the fundings from the Cheung Kong Scholar Program (China), Canada Research Chairs Program and the Natural Sciences and Engineering Research Council of Canada. The earlier version of this paper was presented at the 2006 Stochastic Modeling Symposium of the Canadian Institute of Actuaries. The authors greatly appreciate the constructive comments from the reviewers and participants of the Symposium. They also thank two anonymous referees of the ASTIN Bulletin for their careful reading of the paper and their helpful suggestions that improved the presentation of the paper.

\section{REFERENCES}

Arnold, B.C. (1983) Pareto Distributions. International Co-operative Publishing House, Burtonsville, Maryland.

Artzner, P., Delbaen, F., Eber, J. and Heath, D. (1999) Coherence measures of risk, Mathematical Finance 9, 203-228. 
Assaf, D., Langberg, N., Savits, T., and Shaked, M. (1984) Multivariate phase-type distributions. Operations Research 32, 688-702.

Bowers, N.J., Gerber, H.U., Hickman, J.C., Jones, D.A. and Nesbitt, C.J. (1997) Actuarial Mathematics. Second Edition. The Society of Actuaries, Schaumburg.

CAI, J. (2004) Stop-loss premium. Encyclopedia of Actuarial Science. John Wiley \& Sons, Chichester, Volume 3, 1615-1619.

CAI, J. and LI, H. (2005a) Conditional tail expectations for multivariate phase type distributions. Journal of Applied Probability 42, 810-825.

CAI, J. and LI, H. (2005b) Multivariate risk model of phase-type. Insurance: Mathematics and Economics 36, 137-152.

CEnteno, M.L. (2002) Measuring the effect of reinsurance by the adjustment coefficient in the Sparre Andersen model. Insurance: Mathematics and Economics 30, 37-50.

Centeno, M.L. (2004) Retention and reinsurance programmes. Encyclopedia of Actuarial Science, John Wiley \& Sons, Chichester.

Cossette, H., Gaillardetz, P., Marceau, E., and Rioux, J. (2002). On two dependent individual risk models. Insurance: Mathematics and Economics 30, 153-166

Daykin, C.D., Pentikäinen, T. and Pesonen, M. (1994) Practical Risk Theory for Actuaries. Chapman and Hall, London.

Jorion, P. (2000) Value at Risk: The Benchmark for Controlling Market Risk. Second edition, McGraw-Hill.

Kaas, R., Goovaerts, M., Dhaene, J. and Denuit, M. (2001) Modern Actuarial Risk Theory. Kluwer Academic Publishers, Boston.

Krokhmal P., Palmquist, J., and Uryasev, S. (2002) Portfolio Optimization with Conditional Value-At-Risk Objective and Constraints. The Journal of Risk 4(2), 11-27.

Klugman, S.A., Panjer, H.H. and Willmot, G.E. (2004) Loss Models: From Data to Decisions. Second Edition. John and Wiley \& Sons, New York.

Marshall, A.W. and OlKin, I. (1967) A multivariate exponential distribution. Journal of the American Statistical Association 2, 84-98.

Seal, H. (1969). Stochastic Theory of a Risk Business, John Wiley \& Sons, New York.

WIRCH, J.L. and HARDY, M.R. (1999) A synthesis of risk measures for capital adequacy. Insurance: Mathematics and Economics 25, 337-347.

\section{JUN CAI}

Department of Statistics and Actuarial Science,

University of Waterloo,

Waterloo, Ontario,

Canada N2L $3 G 1$.

Email: jcai@uwaterloo.ca

Ken Seng Tan (Corresponding Author)

Department of Statistics and Actuarial Science,

University of Waterloo, Waterloo, Ontario,

Canada N2L $3 G 1$

and

China Institute for Actuarial Science,

Central University of Finance and Economics,

Beijing, China, 100081.

Email:kstan@uwaterloo.ca 